This item was submitted to Loughborough's Research Repository by the author.

Items in Figshare are protected by copyright, with all rights reserved, unless otherwise indicated.

\title{
A phenomenological interpretation of the parent-child relationship in elite youth football
}

\section{PLEASE CITE THE PUBLISHED VERSION}

http://dx.doi.org/10.1037/spy0000052

\section{PUBLISHER}

(C) American Psychological Association

\section{VERSION}

AM (Accepted Manuscript)

\section{PUBLISHER STATEMENT}

This work is made available according to the conditions of the Creative Commons Attribution-NonCommercialNoDerivatives 4.0 International (CC BY-NC-ND 4.0) licence. Full details of this licence are available at: https://creativecommons.org/licenses/by-nc-nd/4.0/

\section{LICENCE}

CC BY-NC-ND 4.0

\section{REPOSITORY RECORD}

Harwood, Chris G., Nicola J. Clarke, and Christopher J. Cushion. 2019. "A Phenomenological Interpretation of the Parent-child Relationship in Elite Youth Football”. figshare. https://hdl.handle.net/2134/19295. 
Running Head: PARENT-CHILD RELATIONSHIP IN FOOTBALL

A Phenomenological Interpretation of the Parent-Child Relationship in Elite Youth Football

Accepted: Sport, Exercise and Performance Psychology 


\section{Abstract}

2 Youth sport parenting research, in psychology, has methodologically prioritised individual

3 level analysis of the behaviours, perceptions or needs of parents and young athletes. While

4 this has contributed greatly to understanding the role of parents in sport, children's parenting

5 preferences and the challenges of parenting in this unique setting, an exploration of parenting

6 in youth sport from a dyadic, inter-individual perspective has received far less attention.

7 Accordingly, the purpose of this research was to explore parent's and children's experience

8 of their interaction and relationship, in the context of elite youth football. Eight parent-player

9 dyads, recruited from English professional football club youth academies, participated in

10 phenomenological interviews. A two-stage analysis process was performed to explore

11 individual parent and player experiences and examine how accounts related dyadically.

12 Findings present a detailed description and interpretation of the parent-player relationship; as

13 one constituted by relations with other family members, an embodied sense of closeness, the

14 temporal significance of football transitions, and gender relations. This research advocates

15 the need for a view of parenting in youth sport that accounts for how interaction is

16 experienced by both parents and children and highlights the importance of conceptualising

17 parenting as an embodied, temporal process, constituted through interaction and the social

18 context.

19

20 Key words: Parenting, dyadic, youth sport, soccer, qualitative. 


\section{A Phenomenological Interpretation of the Parent-Child Relationship in Elite Youth}

\section{Football}

Parents are a unique influence in the sporting lives of children and an enduring feature of the youth sport landscape. To understand parenting in this context, sport psychology research has predominantly focused on identifying “optimal” parenting involvement to enhance children’s experiences, development and achievement in sport (e.g., Harwood \& Knight, 2015; Knight \& Holt, 2014). Methodologically, studies have prioritised analysis at the individual level - exploring the behaviours, perceptions or needs of parents and/or young athletes. This has contributed greatly to understanding the role of parents in youth sport (e.g., Côté, 1999; Holt, Tamminen, Black, Mandigo \& Fox, 2009; Lauer, Gould, Roman \& Pierce, 2010b), children’s preferences for parenting practices in sport (e.g., Knight, Boden \& Holt, 2010; Knight, Neely \& Holt, 2011; Omli \& Wiese-Bjornstal, 2011) and the complexity and challenges of parenting in this setting (e.g., Clarke \& Harwood, 2014; Dorsch, Smith \& McDonough, 2009; 2015; Wiersma \& Fifer, 2008).

However, an emphasis on analysis at the individual level, risks obscuring an understanding of parenting in youth sport from a dyadic, inter-individual perspective. Dyadic research seeks to understand something of the complex and situated nature of relationships by examining relational phenomena that occur between individuals, such as the content, diversity, quality and pattern of interactions, commitment, conflict and power (Hinde, 1976; 1996). Drawing upon a symbolic interactionist perspective (Blumer, 1969), Poczwardowski, Barott and Jowett (2006) described interpersonal relationships as “a dynamic product of social interaction in which interpretations and meanings are actively negotiated by social actors” (p.130). From this perspective, parenting can be viewed as a dynamic process, constituted through interaction with significant others and the social-cultural context. Research which seeks to explore the social, interpersonal, situated nature of parenting, 
therefore, has the capacity to further contribute to existing knowledge of parenting in youth sport. A focus on an exploration of the parent-child relationship in the context of youth sport, offers one such direction for research.

Social and contextual influences on the parenting process in youth sport have previously been highlighted. For example, Knight and Holt (2013) described how parents’ experiences of watching junior tennis tournaments were affected by children’s performances, on-court behaviours and emotional reactions to matches, alongside the focus on ranking points and organisation of tournaments. Similarly, from longitudinal research with four families over the first 15 months of a child's involvement in organised youth sport, Dorsch et al. (2015) illustrated how parents adjusted their behaviour in response to their child's participation. This interactive process was shaped by the characteristics of the parent, the reciprocal influence of the child and the social context, and supported the authors' previous recommendation; that the quality of the relationship and the sport context in which the relationship exists should be considered in future parenting research (Dorsch et al., 2009). Holt et al. (2009) also analysed interviews with parents and players from the same family, supported by participant observation, to explore the parenting styles and associated practices used by parents of under-12 and under-14 age group female football players. The findings suggested that children reciprocally influenced their parents’ behaviours when they demonstrated responsibility, or challenged unsupportive comments for example. However, this study was limited by interviewing children for the purposes of triangulating parents' descriptions, rather than to represent children's perspectives of parenting. Although these studies have illustrated how the parent-child relationship is shaped by the social context, there is a tendency for findings of this nature to be located within a discussion of the determinants of parents' behaviours and/or associated child effects (e.g., Harwood \& Knight, 2015; Holt et al., 2009; Holt, Tamminen, Black, Sehn \& Wall, 2008). This can isolate parenting from its situated, 
interpersonal context and reduce parent-child relationships to a set of tasks to be achieved (Faircloth, Hoffman \& Layne, 2013; Lee, 2014), if the reciprocal and flexible nature of parenting is not recognised. As Knight and Holt’s (2014) grounded theory of parenting in youth tennis indicated, parents can adapt their involvement over time, dependent on their individual child's needs and goals. Also, the extent to which players perceived that parents understood their tennis journey, the challenges of competing and how sport fitted within their overall lives was a salient feature of children’s experiences.

In addition to the reciprocal nature of parent-child relationships in sport, research using qualitative methods has also begun to describe aspects of these relationships (Clarke \& Harwood, 2014; Dorsch et al., 2009: Kay, 2009; Lauer, Gould, Roman \& Pierce, 2010a). Examining the extent to which fathers can fulfil contemporary expectations of parenting through youth sport, Kay (2009) highlighted that fathers perceived that their son's participation in junior grassroots football provided an opportunity to develop a shared interest and connect emotionally with their sons. Football was an activity through which fathers could become more involved with their son's lives and experience "male bonding” (p.114). As none of the participants had any prior direct involvement with football, Kay (2009) interpreted that fathers became involved through their desire to be a good parent and develop an involved and emotionally close relationship with their son - reflecting the prevalent societal expectations for fathering (Dermott, 2003; Jeanes \& Magee, 2011). This illustrates the influence of cultural constructions of parenting on the meanings parents attach to their relationships with their child.

Supporting the finding that parent-child relationships have the potential to be enhanced through a shared experience of sport, Clarke and Harwood (2014) in their study of parenting experiences in elite youth football, described how the experience of being part of a professional club, sharing a passion for football and travelling to and from matches together 
heightened parents' sense of closeness in their relationship with their son; highlighting the influence of the sport context on parent-child relationships. However, like Kay (2009), this study did not explore whether players had also experienced this increased closeness.

Although the parent-child relationship was not explicitly examined by Dorsch et al., (2009), in group interviews parents described how their involvement in their child's organised team sport programme provided the opportunity for additional and enhanced communication, which led to a perceived higher quality parent-child relationship.

Relationships were described as fluid and dynamic, as friction between parent and child could also be encountered. Similarly, in a retrospective interview study with parent-child dyads, Lauer et al. (2010a) identified that parents facilitated smooth, difficult or turbulent development pathways for elite young tennis players. Although many of the observations made by Lauer et al. focused on parenting behaviours and the impact of these on player outcomes, other findings reflected aspects of the parent-child relationship. For example, the presence of frequent or unresolved conflict (arising from parents' desire to control the tennis experience, or players failing to respond to parents’ advice) led to strained relationships and regret in later years, with some players perceiving that parents' love and support were conditional on tennis success. This underlines the importance of understanding how interpersonal phenomena such as conflict is experienced and negotiated by both parents and players. Exploring parent-child interactions in the present, rather than retrospectively, may also contribute to extending knowledge of this relationship in sport.

In summary, the predominant focus on delineating parenting involvement and associated child outcomes in youth sport through analysis of individual-level constructs, has meant an understanding of parent-child relationships from a dyadic perspective remains a relatively unexplored but potentially fruitful avenue for research. Studies describing aspects of this relationship in sport have drawn attention to the dynamic and complex nature of 
121 parent-child interactions, highlighting that relationship quality, the context in which interactions occur, and both parents' and players’ experiences of interaction such as closeness and conflict should be considered in future research (Clarke \& Harwood, 2014; Dorsch et al., 2009; Kay, 2009; Lauer et al., 2010a) - yet an examination of the parent-child relationship in youth sport was not a specific aim of these studies. Therefore, there is a need firstly to describe the parent-child relationship in detail and to understand how interaction is experienced by both parents and children. Accordingly, the purpose of this research was to explore parent's and children's experience of their interaction and relationship, in the context of elite youth football. English football offers a unique and useful setting in which to study parent-child relationships. Research has begun to illustrate some of the difficulties of parenting in this highly challenging, competitive culture (Clarke \& Harwood, 2014; Harwood, Drew \&

133 Knight, 2010). Becoming a professional footballer is a common aspiration among young 134 players, yet few will progress to be offered a full-time playing contract (Cushion \& Jones, 135 2006). For parents, managing the time and financial commitment required for players to 136 participate in elite training and competition programmes, together with the emotional demands of preparing their child for the potential of deselection, can be a stressful experience 138 (Harwood et al., 2010). Parents of players identified as talented can also experience enhanced status and a heightened responsibility to fulfil societal expectations to facilitate their child's

140 development (Clarke \& Harwood, 2014). Therefore, the high risk high reward nature of elite

141 youth sport can amplify the potential influence of the social context on parent-child 142 relationships.

A phenomenological approach, which is concerned with describing subjective

144 experience and understanding how this is shaped by the social context, offers much potential 145 for exploring relational phenomena such as parent-child relationships, as it foregrounds the 
146 assumption that meaning is constructed between a person and their world, rather than through

147 a cognitive process, or as a response to behaviour (Langdridge, 2008). Furthermore, the

148 phenomenological focus on how the social and material world is experienced as lived (by

149 particular bodies, in particular places, at particular times) allows for an in-depth

150 understanding of how interactions are interpreted by both parents and players. Previous

151 phenomenological studies have described parenting as an embodied, intentional pedagogic

152 relation in which parents orientate towards their child's future and their responsibility of care

153 and children experience a fundamental sense of support and security that empowers them "to

154 be and to become” (van Manen, 1990 p.59). The shared experience of lived time and space

155 can be enriching for parents and children when their reciprocal influence is felt and

156 recognised, but can also lead to vulnerability, when parents experience a lack of control or

157 knowledge of how to act, or children feel parents are unresponsive (De Mol \& Buysse, 2008).

158 This study endeavours to extend existing research by providing a detailed description

159 and interpretation of the parent-child relationship in elite youth football. Specifically, an

160 existential phenomenological approach was selected for this study, guided by Ashworth’s

161 lifeworld analysis (2003a; 2003b). A focus on the phenomenological lifeworld anchors

162 research in understanding everyday lived-experience and explicitly attends to peoples’

163 experiences of relations with others. In addition, this research was informed by van Manen’s

164 (1990) hermeneutic (interpretative) phenomenological approach, to develop a richer

165 understanding of the parent-child relationship. Drawing on Gadamer's (1975) position that

166 "[t]hat which can be understood is language” (p.491), which proposes that being pre-exists

167 language, but is expressed through language, van Manen’s phenomenological project

168 emphasises the role of language in constituting experience. As Langdridge (2007)

169 summarises, “we always speak from somewhere, from a position dependent on our history

170 and culture” (p.42); therefore a focus on language recognises that in dyadic research 
171 interviews participants reflect upon their relations with others, and that this interpretative process is influenced by individuals’ social, cultural and historical backgrounds.

\section{Methods}

\section{Research Context}

In England, the Premier League’s youth football performance pathway is delivered by professional football clubs through their academy programmes. Academies provide programmes of coaching, games, sports science support and education for players across the performance pathway, to “create a fully integrated environment servicing all aspects of the players’ development” (EPPP, 2011 p.18). The pathway comprises three distinct phases; the

180 foundation phase (under-5 to under-11 age group players), the youth development phase

181 (under-12 to under-16 age group players) and the professional development phase (under-17 to under-21 age group players). Clubs can register up to a maximum of 30 players per age group, reducing to 20 in the under-15 and under-16 squads, and 15 in the professional development phase squads (The Football League, 2014). provided with between 12 and 16 hours of evening coaching and weekend competitive matches per week. In addition, in 2011 a hybrid training model was introduced to the academy programme, where young players take time off school to attend daytime training. Players can stay within the academy system for a number of years requiring a significant

190 commitment from players and parents alike. As academy players in the youth development 191 phase can live up to a maximum of 90 minutes travel time from the training ground, parents 192 have an important role to play in transporting their children to training and home matches.

193 Aspiring footballers who successfully progress through the youth development phase will be 194 offered a scholarship to train full-time at the academy for a further two years. At the end of 195 this apprenticeship, a small number of players may be offered a professional playing contract. 
196 The remaining players will be deselected or 'released' from the club. Currently, on average,

197 nine new young players advance from the academy system into first team Premier League

198 football each year (The Football Association, 2014).

\section{Participants}

Ethical approval was obtained from the university’s ethics committee and all participants gave informed consent. Mothers, fathers and players from three English professional football club youth academies were purposively sampled (i.e., had experienced the phenomenon of interest), guided by maximum variation sampling (Patton, 2002).

Variation between participants allows similarities and differences in how a phenomena is experienced to be highlighted (Langdridge, 2007). Therefore, mothers and fathers from one and two-parent families across the youth development phase were recruited to enable a range of parent and player experiences to be examined. Eight parent-player dyads participated in this study; four mothers and four fathers aged between 40 and 49 years $(M=44.75)$, of players aged between 12 and 17 years $(M=14)$. Participants described their ethnicity as White British ( $n=8)$, Black African $(n=2)$, African British $(n=1)$, African Caribbean \&

211 Bengali Spanish $(n=1)$, Spanish Bengali $(n=1)$, White Asian $(n=1)$, White \& Black

212 African $(n=1)$ and White \& Black Caribbean $(n=1)$. Parents self-identified as the person most involved in their son's football participation.

\section{Data Collection}

Dyadic research offers a powerful method for understanding interaction and

216 relationships and has been used extensively within family research (Morgan, Ataie, Carder \&

217 Hoffman, 2013; Wittenborn, Dolbin-MacNab \& Keiley, 2013). Dyadic interviewing, where

218 two people who share a relationship are interviewed separately, allows each person to discuss

219 an experience from their own perspective and also reflect upon their relationship. Interview 220 data can then be explored from an individual and a dyadic perspective, examining overlaps 
and contrasts between accounts (Eisikovits \& Koren, 2010). Using separate dyadic interviews enabled both parent and player experiences of academy football and interaction with the other to be collected.

Participants took part in a phenomenological semi-structured interview, held at parents’ homes or at academy training grounds. Parent and player information sheets and consent forms were sent to participants explaining; the purpose of the research; the format of the interviews; issues pertaining to confidentiality and withdrawal; and how research findings would be used. To ensure players were able to give an informed indication of their willingness to participate, each interview was preceded by a discussion with the researcher to allow participants the opportunity to ask any questions they had. It was emphasised that there was no expectation for them to participate, that consent could be withdrawn at any time, and that participants should consider themselves experts on the research topic. Players were interviewed first, in rooms accessible to parents (or coaches) but where conversations could not be overheard. Four parents also participated in a follow up interview, at the request of the researcher, to discuss certain aspects of their experience more fully. Total interview time was between 33 and 182 minutes $(M=62)$ for players and 40 and 237 minutes $(M=97)$ for parents. The first stage of the player interviews was guided by questions which focused on participants' subjective experiences of playing academy football. The opening question; “tell me about your experience of playing football from when you first began to the present day”, was designed to encourage players to reflect upon and share their experiences in their own words. Subsequent questions explored their present, everyday experiences of playing football. The second stage of the player interviews concentrated on players' experience of interaction with their parents. The purpose of these questions was to prompt players to reflect upon their relationship with their parent, in the context of football. Follow up questions were used to ask players to elaborate and provide more detailed descriptions. Parent interviews 
246 followed a similar format, but rather focused firstly, on their experiences of being a parent of

247 an elite youth footballer and secondly, on their experience of interaction with their son in

248 relation to football. Both interview guides can be found in Appendix A. Interviews were

249 audio recorded and all verbal talk transcribed. Pseudonyms were chosen for each participant

250 and for any person or organisation referred to by name in the interviews to provide

251 confidentiality.

\section{Data Analysis}

Analysis began by reflecting upon each interview directly after it had concluded and audio recording initial impressions and interpretations, which were later used as prompts to write research diary notes. These notes assisted in enhancing reflexivity - a process of "continually reflecting upon interpretations of both our own experience and the phenomena being studied” (Finlay, 2014 p.130) - by checking that initial, instinctive interpretations were not obscuring alternative understandings.

Interview transcripts were explored using the "selective” and "wholistic” reading approaches described by van Manen (1990 p.93), from within a phenomenological attitude, which seeks to set aside taken-for-granted assumptions about the world and remain open to seeing phenomena “afresh” (Finlay, 2014 p.122). Firstly, transcripts were read several times while listening to the respective audio recording, and sections of the text which seemed particularly essential to the participants' individual experience were highlighted, creating meaning units in the data. Descriptive and interpretative notes for each meaning unit were entered into an analysis table, which differentiated between descriptions related to participants' phenomenological lifeworld and to the parent-child relationship. An analysis extract is shown in Appendix B. Next, a wholistic reading of each transcript was used to understand the overall meaning of participants' descriptions and ensure that idiosyncratic 270 interpretations did not move beyond the data. 
A two-stage analysis process was performed to examine how the individual parent

272

273 and player accounts related dyadically. In the first stage, meaning units identified in each interview were interpreted in relation to the seven universal features or 'fractions' of the phenomenological lifeworld as described by Ashworth (2003a; 2003b), paying attention to links between them. This produced a set of thematic interpretations for each individual participant. The seven lifeworld fractions are:

- $\quad$ selfhood (what the phenomenon means for social identity, agency and voice);

- $\quad$ sociality (how the phenomenon affects relations with others);

- embodiment (the role of the body in experiencing the phenomenon, including gender and emotion);

- temporality (the lived-sense of past, present and future);

- spatiality (meanings of space and place in relation to the phenomenon);

- $\quad$ project (how the phenomenon affects people's ability to pursue life activities); and

- discourse (how language is drawn upon to describe the phenomenon).

Secondly, parent and player themes were analysed together by undertaking a wholistic reading (van Manen, 1990) of both transcripts and searching for patterns in the data from a dyadic perspective. Informed by Eisikovits and Koren’s (2010) guidance for dyadic interview analysis, particular attention was paid to overlaps and contrasts in participants' accounts. For example, were experiences described similarly but interpreted differently, or vice versa? In addition, how language was used to construct participants' descriptions was examined closely (in recognition of the constitutive role of language) to avoid uncritically combining individual data and making conclusions about the parent-player relationship. Moving between the dyadic and individual analyses allowed for different interpretations of individual themes and unique relational themes to be constructed.

Drawing upon Gadamer’s (1975) concept that understanding is developed through 
conversation in which we seek a fusion of horizons towards agreement, van Manen (1990) describes his phenomenological approach as a "conversational relation that the researcher develops with the notion he or she wishes to explore and understand” (p.97-98), and emphasises how transforming thematic statements into phenomenologically sensitive writing is a creative, hermeneutic process. Therefore, writing was embraced as an additional method of analysis, in which detailed descriptions of each parent-player relationship were produced from individual and dyadic themes. Extracts of this writing were shared with research peers experienced in qualitative research and knowledgeable of social psychological theories to facilitate further conversation and reflection upon analysis. Collaborators offered additional interpretative lenses which could be used to enhance understanding of participants’ experiences. Finally, the writing and rewriting process (van Manen, 1990) assisted in the production of descriptions which present phenomenological accounts of the parent-player relationship and interprets how this shapes the parenting process.

\section{Findings and Discussion}

Analyses of the participants’ individual and relational experiences are drawn upon to consider the implications for understanding the phenomenology of parent-player relationships in elite youth football. In particular, the findings are interpreted using the phenomenological concepts of relationality, embodiment and temporality, as the lifeworld fractions that appeared most relevant to participants’ experiences (Ashworth, 2003a; 2003b; van Manen, 1990). Although all eight parent-player dyads inform the findings, some more detailed examples are presented, to capture something of the complexity of participants' experiences.

317 The family context for each individual dyad is provided in Appendix C. The findings presented are not claimed to represent a 'true' version of reality and it is acknowledged that other readings of the data are possible and may offer alternative interpretations and understandings of the parent-player relationship. 


\section{Embodiment: Closeness}

Across the parent-player dyads, football was a shared experience which shaped their relationships and was significant in their lives. Players described spending more time with, or feeling closer to their parent through football. This was reflected in parents' accounts, supporting previous findings that parents valued the opportunity to interact more with their child that sport provided (Dorsch et al., 2009; Wiersma \& Fifer, 2008). This closeness in parent-player relationships can be described as a key feature of participants’ livedexperiences, which manifested in various embodied ways. For example, parents experienced excitement from the buzz of the Sunday morning pre-match routine, pride when players felt able to ask for feedback to be given in a different way and uncertainty about the fragility of their son's academy place. Players described feeling frustrated or embarrassed if they did not play well or were substituted when parents were watching, happy when parents gave them positive feedback, and a sense of security from the knowledge that their parent would be there for them if they were released by the club. These emotions can be described as part of the embodied experience of closeness in the parent-player relationship and - following Burkitt's (1999) interpretation of emotions - were constituted through social interaction and shaped by the elite, competitive culture of football academies.

football was particularly pertinent: that's because we don't live together, you know so the time he spends with me is

344 Josh also reflected on the closeness he experienced in his relationship with his father: Author: Do you think football has influenced your relationship with your dad at all? 
Josh: Yeah. It's made us become a lot closer. And cos we both love football it's like we both know what to talk about if you get what I mean so like we have a lot to talk about even though he did not live with his son. In doing so, he met the societal expectations for fathers to have an active role in parenting and to develop open, close relationships with their children (Dermott, 2003; Jeanes \& Magee, 2011). Having a high-quality father-child relationship has also been associated with lower psychological distress in non-resident fathers (Vogt Yuan, in press). This research, therefore, supports the claim that youth sport provides a setting where fathers can enact involved parenting (Coakley, 2006; Kay, 2009; Trussell \& Shaw, 2012) and furthermore, that this can influence perceptions of relationship quality, which may be salient to non-resident fathers. understanding of how football influenced players' everyday subjective experience. Luke, who was in his first season of balancing playing football full-time with his education, described feeling closer to his father than his siblings were, because after his parents separated he continued to see him more at football. Football was something he and his father talked about frequently and had "always worked towards”; indicating that they shared a mutual understanding of Luke's goal of becoming a professional footballer. This influenced Luke’s decision to move in with his father, as he found balancing his educational and football 
371

372

373

374

375

376

381

382

383

384

385

386

387

388

389

390

391

392

393

394

395

commitments challenging and wanted to have someone there to push him to "stay on top of everything”. His father was uniquely positioned to be aware of and understand his life as a whole. Moreover, he reflected that he did not think he would be where he was right now if he did not live with his father. Similarly, his father recognised that he had spent more time with Luke than his other children due to football, but that this was justified because of Luke’s success, which he perceived he would not have been able to achieve without his support. Mike: It has meant that I have spent a ridiculous amount of time with him as opposed to the other two. And I probably would have spent more time with the other two if I wasn't dragged away all around the country with him. However he would have never have gotten the level of support he probably needs and needed had I have not been able to sacrifice the time to do that

Illustrating an overlap in their accounts, Mike constructed his involvement as unique, by suggesting that Luke would "never” have received this support otherwise, because of its sacrificial nature; and by emphasising the permanence and necessity of his support to meet his son's needs. Likewise, Luke interpreted that his father "knows me better than anybody else”, in particular with regards to football, as whereas his coaches and teammates had changed, his father had been "the only person that's been with me the whole time". This reflects how a shared understanding of his everyday subjective experience was an important aspect of Luke's relationship with his father, which contributed to the sense of closeness in the relationship.

This finding indicates that the elite culture of football academies, which demands high levels of commitment and involvement from players and parents over a prolonged period of time, can uniquely contribute to increased closeness in the parent-child relationship. The shared corporeal understanding of players' football experience, and how this fits in with their lives, also enhanced this closeness and supports Knight and Holt’s (2014) postulation that 
developing an understanding emotional climate is integral to parents enhancing their child's tennis journey. The experience of closeness enabled fathers to participate in involved fathering, however mothers described feeling closer to their son despite a perceived lack of football knowledge, and is described below.

\section{Embodiment: Gender}

Exploring both mother- and father-player dyads highlighted the gendered dimension of parent-child relationships. Professional football clubs are controlled and organised by men and permeated by dominant masculine norms and values (Roderick, 2006). Youth sport can provide a setting where fathers feel comfortable and competent to perform parenting, as men’s knowledge and experience of sport are considered authoritative (Coakley, 2006; Willms, 2009). How mothers experience this subordination is unclear, yet mothers must also negotiate contemporary cultural expectations of intensive mothering (child-centred, expertguided, emotionally absorbing and time and labour intensive) when supporting their son's sport participation (Hays, 1996; Trussell \& Shaw, 2012). For example; Carla, a single mother to Ali, experienced uncertainty about the effectiveness of her feedback to her son. Feeling that she was "constantly on his case” to keep him motivated in football and in school meant she questioned the degree to which she pushed her son and the effectiveness of her advice as a mother. I do wonder you know am I just a nag, does he just think I’m a nag. [ ] A boy learns certain things from his father and if he's not there, and there isn't another man in the house, where does that come from? I mean I've been taking Ali to football since he was five years old it's always been me. And I've always been the one giving him the advice and everything, and I love football. I've been watching it- I watched it with my older brother since I was a young girl so I kind of know what I'm talking about! But 
obviously you have the coaches teaching them whatever they teach them, and I have said to Woody and Wrighty in the meeting, sometimes I don't know whether what I say to him is a help or a hindrance

Despite this uncertainty, Ali praised his mother's parenting approach and described feeling closer to his mother than his father (who separated from his mother when he was very young) because of the time they had spent together through football.

Ali: She like helps me analyse the game after sometimes, she makes sure I'm not too hard on myself, like I don’t beat myself up over mistakes, she always encourages me on the sideline tells me what I'm not doing enough of, stuff like that Author: And is that helpful having that feedback from your mum? Ali: Yeah it's quite good yeah so coz I can't watch myself, coz they record it but I don’t- we don’t get to see it, they just watch it. But yeah she just tells me what I do really, and if I can do better or anythin

In this extracts Ali describes how he accepts his mother's advice because he interprets that it has a positive impact on him. This is in contrast to Carla's anxiety that her guidance was construed as "nagging” by her son and represents a divergence in their individual accounts. Her embodied sense of interaction with his son - that her feedback as a mother was not effective - illustrates the gendered nature of parenting experiences. By positioning herself within this gender order - the pattern of power relations between men and women (Connell, 1987) - Carla experienced greater uncertainty and questioned the value of her advice to her son in this setting, even though at other points in the interview she described her detailed knowledge of and love for football.

However, the value Ali placed on his mother's advice after football matches was in contrast to many of the players whose fathers were identified as the most involved in their football. Players often described their mother's role in limited terms because of a perceived 
lack of football knowledge.

Author: Is your mum involved in football at all?

Josh: She’s supportive, she’s like, she don’t know much about football at all. She just says like enjoy it and stuff, so she’s not really like- she wouldn't watch football or like she wouldn't- she don’t really know much about football basically heh. So it's hard to get something from her

Jason: My stepdad gets more sort of involved sort of thing than my mum because he knows a bit more about football than her, and like is more interested and stuff like that Perceived lack or undervaluing of football knowledge was also reflected in the interviews with parents. Mothers described how their understanding of football developed a sense of closeness with their son, but typically downplayed their level of knowledge. Steph: Because obviously I'm a female, being able to enjoy that with them has helped our relationship because he's so into it. I suppose I've got enough football knowledge and like of the game enough to you know to be able to enjoy that with him

Susan: I think if Jason hadn't have done the football in the way that he did I would struggle to have conversations with them about anything sometimes. It is a topic of conversation I can now join in, and not feel I don't know anything about it. I don’t know as much as everybody else but I feel as though I'm part of it These findings imply that knowledge and understanding of football was assumed and accepted with fathers or step-fathers, but not mothers, reflecting the dominant masculine norms and values of professional football (Roderick, 2006). This supports and extends Holt et al.'s (2008) finding that perceived knowledge and experience influenced parents' involvement at youth sport competitions, by highlighting how certain knowledge is 
471 authoritative within sport cultures. Importantly, perceived knowledge also has the potential to 472 contribute to an embodied sense of closeness in the parent-child relationship, which may be 473 problematic for mothers who lack or devalue their experiential knowledge of football. In the 474 above quotes, although mothers consistently downplayed their knowledge, they emphasised 475 how this understanding enhanced their relationship and improved communication with their sons, highlighting a unique aspect of the parent-child relationship in this setting.

\section{Temporality and Transitions}

Interaction between parent and player was influenced by the temporal significance of the players' next transition in football. Transition points occurred towards the end of each season when a decision would be made by the club as to whether to extend players' contracts. Players described experiencing pressure to develop and impress coaches within a definitive timeframe, or else their academy status would be at risk. Likewise, parents recognised that their son's place in the academy was not secure and were aware of the restricted period of time in which their child had to meet expected performance standards.

At 15 years old, and playing in the under-16 squad, Harry would learn within the next month whether the club would offer him a scholarship to play full-time academy football. The imminent scholarship decision created a shared experience of uncertainty for father and son. Harry described feeling nervous about the impending decision but also a sense of assurance that he would be able to play at another club in a lower tier of English football if he was not offered a scholarship at his current academy. He described being unconcerned about looking for another club, as his aim was simply to play football. This was in contrast to his father Steve, who experienced increased anxiety about his son’s future at the club and a desire for a resolution. 
blah. I’ve been quite laid back about the whole thing really over the years, now even I'm starting to feel it a little bit. I'm getting worried f'him if he has a bad game it's like ohh you know hope they're not gonna drop him. You do start getting like that. I just want it to be over really I just want to get him sorted

For Steve, success was judged by whether or not Harry was offered a professional contract. His concern that the club might "drop" his son and his desire to "get him sorted", alongside the prominence of football in his family’s life; “football’s just a life for us it’s a lifestyle”, implies that Steve's experience of pressure was constituted through his connection to his son's football participation, and that his identity was in part constructed through Harry’s achievements in football. Furedi (2002 p.107) suggested that “adults do not simply live their lives through their children, but in part, develop their identity through them”. In the sport domain, Smoll, Cumming and Smith (2011 p.16) termed this experience "reverseddependency”, where parents define their own self-worth in terms of their child's sporting successes or failures. Steve's shared football identity with his son meant that he was committed to supporting his son's pursuit of a professional football playing career. Smoll et al. (2011) assumed that reverse-dependency was accompanied with excessive parental pressure. However, this was not apparent in Harry’s interpretation of his interaction with his father. He acknowledged his father's determination "to get him being a footballer”, but rather than this expectation increasing the sense of pressure to succeed, he interpreted his parent's behaviours in relation to achieving his personal goal of playing professional football. Author: And in what ways have they helped you Harry: Well, took me training that's the main part. They tell me what I need to improve on, like in the nicest way and then they tell me like how I can improve it. So dad got me a personal trainer, which helped me a lot. I wouldn't have been able to get myself a personal trainer, so they get a lot of stuff like that 
Author: And you say they tell you stuff in the nicest way what does that mean Harry: Uhh they like, they just say ohh you need to work on your speed a little bit, I've just seen like an example and then they give me an example of why and I'm like yeah I do. And then they give me advice on how to do it, like my dad will take me out training, speed training. So they don't just tell me 'yeah you need to work on your speed or you won’t get a scholarship’

Here, Harry described accepting feedback from his father (and grandfather) on how to improve, explaining how it was delivered in a supportive way. By using "just” and "a little bit” to describe how advice was typically given, he minimised the authoritarian nature of their instruction. Instead, he constructed this feedback as based on evidence which he agreed with, and further described how his father was then actively involved in helping him practise at home. In doing so, Harry positioned himself as an active agent in the process of assessing his weaknesses, as opposed to a passive recipient of evaluation and corrective instruction. His reference to a failure to obtain a scholarship alluded to a shared understanding of the potential negative consequence for not undertaking additional physical training, but dismissed that his father used this as a way of exerting control.

However, whereas Steve interpreted Harry’s enthusiasm for training to mean that he was playing without the pressure he experienced so intensely himself, Harry described how he completed additional training and fitness because "if the coaches know that you are doing extra work they will like you a lot more because you are determined to get better”, suggesting that Harry was motivated to follow his father's instruction in order to increase his chances of being offered a scholarship. Interpreting his interaction with his father in this way indicates how the temporal significance of transition points in football influenced their relationship. Similarly, across the dyads, this sense of lived-time in relation to their son's future reinforced parents’ commitment to facilitate their son’s football development and was used to 
546 justify certain practices, for example criticising performances. Criticism was legitimised as a

547 practice necessary for preparing their son for a future in professional football:

Ade: It's good that I tell him already now and then I'm harsh with him and then because he wants to become professional footballer he's prepared to take whatever I'm going to throw to him, umm because I know after this it's gonna be tough. It's gonna be very bad

This reflects Jeanes and Magee’s (2011) finding that parenting practices which contradict the orthodox expectations of involved fathering, such as aggressive or controlling behaviours, were rationalised by fathers (of 14-year-old academy players) as necessary for equipping their sons for the masculine, competitive, elite environment of professional football.

Although criticising performances was a strategy used by some fathers in the present study, in the face of temporal uncertainty parents also described complying with academy expectations (e.g., to attend additional training), proactively making contact with other clubs (a practice discouraged by the Premier League), and emphasising the importance of education to manage their son's approaching transition point. concerned, it's more important. If the football works out that's fabulous and you know you're going to have plenty of money, that'd be great, but the likelihood is that's not going to happen. And you have got to do your schoolwork because this is Natasha: I don’t want him to be broken hearted if he doesn't, because he might not fit your opportunity to get your schoolwork right in here but he might fit in somewhere else. There's other kids that have left that have fitted in other places so, we just try and say that to him and he's fine. And he even in the car he was saying about school, I heard him and his friend, ‘you've gotta make 
sure you do well at school because you've gotta have a backup plan.' I'm thinking good that's good because that's what we try and say make sure you do well at school because you could be injured, you could, anything could happen. You can’t count on it

These findings indicate how the temporal nature of parenting experiences in football compelled parents to act in ways that accepted or resisted the academy's control, mirroring the experiences of parents of foundation phase academy players (Clarke \& Harwood, 2014). This represents a unique aspect of parent-child relationships in this setting. As other phenomenological studies have illustrated, parents and children experience a shared sense of lived-time, in which parents are future-orientated and hopeful for their child's becoming (De Mol \& Buysse, 2008; van Manen, 1990). In elite youth football, this temporality is experienced differently, as fixed timescales and arbitrary decisions for transition points threaten parents and players’ shared identity, goals and future possibilities for relational experiences.

\section{Relations with Family}

The experiences of parents and players in this study highlight how parent-child interaction occurred within the context of wider, more complex family relationships. A variety of individual adults may be involved in the care of children including step-parents, grandparents, older siblings and other family members (Ribbens McCarthy \& Edwards, 2011). Each dyad in this study was part of a unique network of family relationships, which shaped their interaction in different ways. For example, Harry's grandfather was considerably involved in his football. He provided transport and technical advice to his grandson, and monitored his performance to pass on information to Harry's father. The regret that Harry's father experienced because his own father did not allow him to sign a contract with a professional club also influenced his decision to support Harry to play academy football. At 
59615 years of age he was offered a youth contract by the same club that his son now plays at, 597 but was not allowed to sign it because his own father decided “it was too one-sided”. He 598 regrets that he was denied the chance of playing higher level football and wonders what he 599 could have achieved as a player if he had signed to the club. When his son started playing 600 football he discussed with his father that if Harry was offered a similar opportunity he would 601 let him sign a contract, highlighting how his parenting was influenced by his personal experiences with his father. players’ own relationship with their parent. For example, having two older brothers who had been through the academy system meant for Theo that he anticipated how his interaction with his father was likely to change: Theo: I guarantee if I stay until the under 13s, he will talk a lot and a lot. He talks a lot to my brother, like just gives him a conversation for like an hour. For me he speaks like 20 minutes, but when I get older probably be an hour as well. It's long.

His father Ade also acknowledged that his approach was influenced by his experience of supporting his other sons' football participation. Describing professional football as "very ruthless and very cruel”, he had resolved to "never be laidback again” and instead, be more involved in facilitating opportunities for his youngest son to play and progress. professional footballer I know what it takes, what you need to have. So that's the bottom line. positively support the development of young athletes when brothers and sisters are cooperative rather than competitive (Côté, 1999), that parents can struggle balancing time 
621 with their other children (Harwood et al., 2010; Harwood \& Knight, 2009a; 2009b) and that sport can act as a catalyst for changing the dynamics of family interaction (Dorsch et al.

623 (2015). The interdependence of family relationships in sport, therefore, remains an important 624 area of interest for future research.

625 Furthermore, this study lends support to a family systems approach to research and to 626 working with families to enhance experiences in sport. Family systems theory, although 627 typically used in family therapy, has been utilised in the sport setting and focuses on understanding individuals in the context of their relationships with family members

629 (Hellstedt, 2005; Zito, 2010). This approach aims to develop the necessary support for

630 families to manage change and adapt to the transitions that children may experience as they

631 progress in sport, by identifying patterns of functional or dysfunctional interaction. This

632 offers an alternative intervention strategy to types of parental education programmes which

633 seek to increase parents' knowledge and awareness through a “one-size-fits-all” solution to

634 optimising parental involvement in sport, which often fails to consider the dynamic and

635 varied nature of family relationships (Holt \& Knight, 2014 p.114). However, family systems

636 theory is limited by underlying assumptions which determine what is meant by functional

637 interaction within a family, and a failure to account for the influence of political, social and 638 economic power in relationships (Walters, 1990). Therefore, future research which explores

639 parent-child relationships in the context of the family - including individuals who a child

640 identifies as having a significant relationship with - can further enhance understanding of the

641 complex process of parenting in sport; especially when the influence of gender relations and

642 temporal transitions within the sport culture are considered.

\section{Conclusions and Future Directions}


646 youth football. The findings present a detailed description and interpretation of the parent647 player relationship; as one constituted by relations with other family members, an embodied sense of closeness, the temporal significance of football transitions, and gender relations. It is suggested that these experiential aspects may serve as a useful heuristic (although not

650 foundational) guide for researchers and practitioners working with families to encourage

651 reflection on current understandings of parent-child relationships and interaction in this context.

Uniquely to elite youth football, parents and players experienced increased closeness

654 through the shared and embodied experience of academy football and a heightened sense of

655 lived-time as families prepared for the player's next transition point and the potential

656 consequences of academy decisions. The corporeal understanding of players' football

657 experience and how this fitted in with children's lives, developed over time through interaction, was valued by players. However, the constitutive role of gender to the parentchild relationship represents an area which requires further exploration. As De Mol and

660 Buysse (2008) highlighted, parents can experience vulnerability when they lack control or 661 knowledge of how to act toward their child. Mothers in the male dominated environment of 662 elite youth football frequently devalued or questioned their knowledge of the game. Further research which examines the power of gender relations to enable or restrict parenting experiences is required in order to challenge assumptions about what constitutes authoritative

665 knowledge within sport cultures. In addition, the finding that parent-child relationships

666 shaped, and were shaped by, relations with other family members has implications for future 667 research; in particular that examining parent-child relationships in isolation from the family 668 milieu may limit understanding of the parenting process. A limitation of the present study, 669 therefore, was the focus on the experiences of biological parents, which may have excluded 670 step-parents or grandparents who were actively involved in parenting and with players' 
671 football.

672 By illustrating how players experienced agency in the parenting process, this research

673 also emphasised how children mutually constitute their relationship with parents. For

674 example, rather than the experience of reverse-dependency (where parents define their self-

675 worth in terms of their child's sporting success or failure; Smoll et al., 2011) being

676 interpreted as pressurising, players made sense of parenting behaviours in relation to

677 achieving their personal goal of playing professional football, when they felt their aspirations

678 were shared by their parent. This supports previous research that shared and communicated

679 goals for youth sport can enhance parent-child interactions (Harwood \& Knight, 2015;

680 Knight \& Holt, 2014) and advocates the need for a view of parenting that accounts for how

681 relationships are experienced by parents and children, rather than a sole focus on explicating

682 optimal parental involvement. Similarly, parents' lived-experience of developing and acting

683 upon a shared understanding of their child's identity, goals and everyday experiences to

684 effectively manage and negotiate transitions in sport, presents an alternative to the

685 construction of parenting as a skill set which parents must learn to be effective. Harwood and

686 Knight (2015, p.26) proposed that self-development to "learn the trade” of being a sport

687 parent is required for parents to be able to offer appropriate types of social support. This,

688 however, does not recognise parents’ ability to empathise with their child's lived-experience

689 and that this understanding may enable parents to provide social support which is valued by

690 their child. Parents' recognition and understanding of their child's needs and their capacity to

691 adapt to meet these has previously been reported (e.g. Dorsch et al., 2009; 2015, but is often

692 overshadowed within wider discussions focusing on the determinants of parents' behaviours

693 and/or associated child effects. For example, parents’ empathy with their child has been

694 framed as a contributory factor to parents' emotional reactions at competitions, and therefore,

695 as a potential area of development (Holt et al., 2008; Knight \& Holt, 2013). Considering the 
emphasis on the need for parent education in the sport psychology literature (e.g., Gould,

697 Lauer, Rolo, Jannes \& Pennisi, 2008; Knight et al., 2011; Wiersma \& Fifer, 2008), further

698 research to establish the circumstances in which expert advice in elite youth sport is

699 legitimate and warranted, or when active support for parental instinct and judgement can be

700 encouraged may therefore be useful. Furthermore, the temporal uncertainty experienced by

701 parents and the constitutive role of transitions points to parent-child relationships suggest that

702 parents' need for expert advice and/or active support may vary depending on their child’s

703 development stage in football. Academies and practitioners may wish to reflect upon how

704 best to meet this need to ensure families are prepared to manage players’ transitions.

705 In conclusion, by illustrating a fraction of the complex, dynamic nature of parent-

706 child relationships in the context of youth football, this research has highlighted the

707 importance of conceptualising parenting as embodied, temporal process, constituted through

708 interaction and the social context. Further research which aims to achieve a detailed

709 description of these relationships has the potential to contribute to a base of knowledge, from

710 which researchers can build theory and attempt to explain this ubiquitous sporting

711 phenomenon.

\section{References}

713 Ashworth, P. (2003a). An approach to phenomenological psychology: The contingencies of

714 the lifeworld. Journal of Phenomenological Psychology, 34(2), 145-156.

715 Ashworth, P. (2003b). The phenomenology of the lifeworld and social psychology. Social

$716 \quad$ Psychological Review, 5(1), 18-34.

717 Blumer, H. (1969). Symbolic interactionism. Los Angeles, CA: University of California

$718 \quad$ Press.

719 Burkitt, I. (1999). Bodies of thought. London: Sage Publications Ltd.

720 Clarke, N. J., \& Harwood, C. G. (2014). Parenting experiences in elite youth football: A 
phenomenological study. Psychology of Sport and Exercise, 15(5), 528-537. doi:10.1016/j.psychsport.2014.05.004

723

724

725

726

727

728

729

730

731

732

733

734

735

736

737

738

739

740

741

742

743

Coakley, J. (2006). The good father: Parental expectations and youth sports. Leisure Studies, 25(2), 153-163. doi:10.1080/02614360500467735

Connell, R. W. (1987). Gender and power: Society, the person and sexual politics. Cambridge: Polity Press.

Côté, J. (1999). The influence of the family in the development of talent in sport. The Sport Psychologist, 13(4), 395-417.

Cushion, C., \& Jones, R. L. (2006). Power, discourse, and symbolic violence in professional youth soccer: The case of Albion football club. Sociology of Sport Journal, 23(2), 142161.

De Mol, J., \& Buysse, A. (2008). The phenomenology of children's influence on parents. Journal of Family Therapy, 30(2), 163-193. doi:10.1111/j.1467-6427.2008.00424.x

Dermott, E. (2003). The 'intimate father': Defining paternal involvement. Sociological Research Online, 8(4). Retrieved from http://www.socresonline.org.uk/8/4/dermott.html. doi:10.1080/2159676X.2013.857708

Dorsch, T. E., Smith, A. L., \& McDonough, M. H. (2015). Early socialization of parents through organized sport. Sport, Exercise and Performance Psychology, 4(1), 3-18.

Dorsch, T. E., Smith, A. L., \& McDonough, M. H. (2009). Parents' perceptions of child-toparent socialization in organized youth sport. Journal of Sport and Exercise Psychology, 31(4), 444-468.

Eisikovits, Z., \& Koren, C. (2010). Approaches to and outcomes of dyadic interview analysis. Qualitative Health Research, 20(12), 1642-1655. doi:10.1177/1049732310376520

Faircloth, C., Hoffman, D.M., \& Layne, L. L. (2013). Parenting in global perspective. 
Abingdon: Routledge.

Finlay, L. (2014). Engaging phenomenological analysis. Qualitative Research in Psychology, 11(2), 121-141. doi:10.1080/14780887.2013.807899

Furedi, F. (2002). Paranoid parenting: Why ignoring the experts may be best for your child. Chicago: Chicago Review Press.

751

752

753

754

755

756

757

758

759

760

761

762

763

764

765

766

767

768

769

770

Gadamer, H-G. (1975). Truth and method. (J. Weinsheimer \& D. G. Marshall, Trans). London: Bloomsbury Academic.

Gould, D., Lauer, L., Rolo, C., Jannes, C., \& Pennisi, N. (2008). The role of parents in tennis success: Focus group interviews with junior coaches. The Sport Psychologist, 22(1), 18-37.

Harwood, C. G., \& Knight, C. J. (2015). Parenting in youth sport: A position paper on parenting expertise. Psychology of Sport and Exercise, 16(1), 24-35. doi:10.1016/j.psychsport.2014.03.001

Harwood, C., \& Knight, C. (2009a). Stress in youth sport: A developmental investigation of tennis parents. Psychology of Sport and Exercise, 10(4), 447-456. doi:10.1016/j.psychsport.2009.01.005

Harwood, C., \& Knight, C. (2009b). Understanding parental stressors: An investigation of British tennis-parents. Journal of Sports Sciences, 27(4), 339-351. doi:10.1080/02640410802603871

Harwood, C., Drew, A., \& Knight, C. (2010). Parental stressors in professional youth football academies: A qualitative investigation of specializing stage parents. Qualitative Research in Sport and Exercise, 2(1), 39-55. doi:10.1080/19398440903510152

Hays, S. (1996). The cultural contradictions of motherhood. New Haven: Yale University Press.

Hellstedt, J. (2005). Invisible players: A family systems model. Clinics in Sports Medicine, 

24(4), 899-928. doi:10.1016/j.csm.2005.06.001

772

773

774

Hinde, R. A. (1976). On describing relationships. Journal of Child Psychology and Psychiatry, 17(1), 1-19. doi:10.1111/j.1469-7610.1976.tb00370.x

Hinde, R. A. (1996). Describing relationships. In A. E. Auhagen, \& M. Salish (Eds.), The diversity of human relationships (pp. 7-35). Cambridge: Cambridge University Press.

Holt, N. L., \& Knight, C. J. (2014). Parenting in youth sport: From research to practice. Abingdon: Routledge.

Holt, N. L., Tamminen, K. A., Black, D. E., Mandigo, J. L., \& Fox, K. R. (2009). Youth sport parenting styles and practices. Journal of Sport and Exercise Psychology, 31(1), 3759.

Holt, N. L., Tamminen, K. A., Black, D. E., Sehn, Z. L., \& Wall, M. P. (2008). Parental involvement in competitive youth sport settings. Psychology of Sport and Exercise, 9(5), 663-685. doi:10.1016/j.psychsport.2007.08.001

Jeanes, R., \& Magee, J. (2011). Come on my son! Examining fathers, masculinity and 'fathering through football'. Annals of Leisure Research, 14(2-3), 273-288. doi:10.1080/11745398.2011.616483

Kay, T. (2009). Fathers and sons: Being 'father angel'. In Kay, T (Ed.), Fathering Through Sport and Leisure (pp. 106-123). Abingdon: Routledge.

Knight, C. J., \& Holt, N. L. (2013). Factors that influence parents' experiences at junior tennis tournaments and suggestions for improvement. Sport, Exercise, and Performance Psychology, 2(3), 173-189.

Knight, C. J., \& Holt, N. L. (2014). Parenting in youth tennis: Understanding and enhancing children’s experiences. Psychology of Sport and Exercise, 15(2), 155-164. doi:10.1016/j.psychsport.2013.10.010

Knight, C. J., Boden, C. M., \& Holt, N. L. (2010). Junior tennis players’ preferences for 
parental behaviors. Journal of Applied Sport Psychology, 22(4), 377-391. doi:10.1080/10413200.2010.495324

798

799

800

801

802

803

804

805

806

807

808

809

810

811

812

813

814

815

816

817

818

819

820

Knight, C. J., Neely, K. C., \& Holt, N. L. (2011). Parental behaviors in team sports: How do female athletes want parents to behave? Journal of Applied Sport Psychology, 23(1), 76-92. doi:10.1080/10413200.2010.525589

Langdridge, D. (2007). Phenomenological psychology: Theory, research and method. Harlow, Essex: Pearson Education Ltd.

Langdridge, D. (2008). Phenomenology and critical social psychology: Directions and debates in theory and research. Social and Personality Psychology Compass, 2(3), 1126-1142. doi:10.1111/j.1751-9004.2008.00114.x

Lauer, L., Gould, D., Roman, N., \& Pierce, M. (2010a). How parents influence junior tennis players development: Qualitative narratives. Journal of Clinical Sport Psychology, 4(1), 69-92.

Lauer, L., Gould, D., Roman, N., \& Pierce, M. (2010b). Parental behaviors that affect junior tennis player development. Psychology of Sport and Exercise, 11(6), 487-496. doi:10.1016/j.psychsport.2010.06.008

Lee, E. (2014). Experts and parenting culture. In E. Lee, J. Bristow, C. Faircloth, \& J. Macvarish, Parenting culture studies (pp. 51-75). Basingstoke, Hampshire: Palgrave Macmillan.

Morgan, D. L., Ataie, J., Carder, P., \& Hoffman, K. (2013). Introducing dyadic interviews as a method for collecting qualitative data. Qualitative Health Research, 23(9), 12761284. doi:10.1177/1049732313501889

Omli, J., \& Wiese-Bjornstal, D. (2011). Kids speak: Preferred parental behavior at youth sport events. Research Quarterly for Exercise and Sport, 82(4), 702-711.

Patton, M. Q. (2002). Qualitative research and evaluation methods ( $3^{\text {rd }} \mathrm{ed}$.). Thousand Oaks, 

CA: Sage Publications Ltd.

822

823

824

825

826

827

828

829

830

831

832

833

834

835

836

837

Poczwardowski, A., Barott, J. E., Jowett, S. (2006). Diversifying approaches to research on athlete-coach relationships. Psychology of Sport and Exercise, 7(2), 125-142. doi:10.1016/j.psychsport.2005.08.002

Ribbens McCarthy, J., \& Edwards, R. (2011). Key concepts in family studies. London: Sage Publications Ltd.

Roderick, M. (2006). The work of professional football: a labour of love? Abingdon: Routledge.

Skinner, E., Johnson, S., \& Snyder, T. (2005). Six dimensions of parenting: A motivational model. Parenting: Science and Practice, 5(2), 175-235. doi:10.1207/s15327922par0502_3

Smoll, F. L., Cumming, S. P., \& Smith, R. E. (2011). Enhancing coach-parent relationships in youth sports: Increasing harmony and minimizing hassle. International Journal for Sports Science and Coaching, 6(1), 13-26. doi:10.1260/1747-9541.6.1.13

The Football Association. (2014, May). The FA chairman's England commission report.

The Football League. (2014, August). Charter for academy players and parents 2014/15.

The Premier League. (2011, May). Elite player performance plan.

Trussell, D. E., \& Shaw, S. M. (2012). Organized youth sport and parenting in public and private spaces. Leisure Sciences: An Interdisciplinary Journal, 34(5), 377-394. doi:10.1080/01490400.2012.714699

van Manen, M. (1990). Researching lived experience: Human science for an action sensitive pedagogy. New York: State University of New York Press.

Vogt Yuan, A. S. (In press). Father-child relationships and nonresident fathers’ psychological distress: What helps and what hurts? Journal of Family Issues.

doi:10.1177/0192513X14526394 
846 Walters, M. (1990). A feminist perspective in family therapy. In R. J. Perelberg, \& A. C. 847 Miller (Eds.), Gender and power in families (pp. 13-33). London: Karnac Books. 848 Wiersma, L. D., \& Fifer, A. M. (2008). "The schedule has been tough but we think it's worth 849 it": The joys, challenges, and recommendations of youth sport parents. Journal of $850 \quad$ Leisure Research, 40(4), 505-530.

851 Wittenborn, A. K., Dolbin-MacNab, M. L., \& Keiley, M. K. (2013). Dyadic research in 852 marriage and family therapy: Methodological considerations. Journal of Marital and 853 Family Therapy, 39(1), 5-16. doi:10.1111/j.1752-0606.2012.00306.x

854 Zito, M. (2010). Family systems interventions in sport. In S. Hanrahan \& M. B. Anderson 855 (Eds.), Routledge handbook of applied sport psychology (pp. 177-185). Abingdon: $856 \quad$ Routledge. 